INPLASY

PROTOCOL

To cite: Shulhub et al. Minimal important differences in hearing disorder studies: $A$ scoping review protocol. Inplasy protocol 202170048. doi:

10.37766/inplasy2021.7.0048

Received: 15 July 2021

Published: 16 July 2021

Corresponding author:

Abdullah Bin Shulhub

ashalhoob@ksu.edu.sa

Author Affiliation:

University of Manchester.

Support: None.

Review Stage at time of this submission: Preliminary

searches.

Conflicts of interest:

None declared.

\section{Minimal important differences in hearing disorder studies: A scoping review protocol}

Shulhub, AB'; Almufarrij, I2; Munro, KJ33.

Review question / Objective: The objective of this study is to identify and catalogue studies that have included MIDs among the outcome measures used in interventions for adults with a hearing disorder.

Condition being studied: Minimal important differences (MIDs) are the smallest difference in the outcome of interest that patients or informed proxies perceive as important (either beneficial or harmful) and would lead the patient or clinician to consider a change in management. Despite their importance, there is a dearth of hearing disorder studies investigating MIDs. The aim of this scoping review is to identify and catalogue the evidence for MIDs in interventions for adults with hearing disorders.

INPLASY registration number: This protocol was registered with the International Platform of Registered Systematic Review and Meta-Analysis Protocols (INPLASY) on 16 July 2021 and was last updated on 02 August 2021 (registration number INPLASY202170048).

\section{INTRODUCTION}

Review question / Objective: The objective of this study is to identify and catalogue studies that have included MIDs among the outcome measures used in interventions for adults with a hearing disorder.
Rationale: A few studies have measured minimal important differences (MIDs) in adults with hearing disorders (e.g., McShefferty et al. [2016]), but this appears to be relatively unusual. Identifying studies that have assessed MIDs would be beneficial for further research because the MID could be documented and the 
methods used to determine them could be used in future studies that measure MID. Determining MIDs will inform future intervention studies for adults with hearing disorders. To date, no scoping review has investigated the use of MIDs in adults with hearing disorders (i.e. hearing loss, tinnitus and hyperacusis).

Condition being studied: Minimal important differences (MIDs) are the smallest difference in the outcome of interest that patients or informed proxies perceive as important (either beneficial or harmful) and would lead the patient or clinician to consider a change in management. Despite their importance, there is a dearth of hearing disorder studies investigating MIDs. The aim of this scoping review is to identify and catalogue the evidence for MIDs in interventions for adults with hearing disorders.

\section{METHODS}

Search strategy: This scoping review will employ a three-step search strategy to identify published papers. - Step 1: PubMed will be searched using keywords and Medical Subject Headings (MeSH) terms that are relevant to this review (e.g., 'hearing disorder [i.e. HL, tinnitus and hyperacusis]' and 'minimally important difference'; see Appendix I) to identify articles. - Step 2: The medical information specialist changed the query and applied it to the Web of Science, Psyclnfo, EMBASE, EMCARE, Cochrane Library and Academic Search Premier databases after the PubMed search. The terms (minimal clinically important difference AND hearing loss) were used in Google Scholar to compile a complete list of existing articles to identify previously unrecognised publications (see Appendix II). - Step 3: Additional reference and citation searches will also be conducted. The references lists of the articles identified during the search will be scanned manually. If possible, citations of selected articles by other scholars will be searched using Google Scholar's 'cited by' feature to identify any relevant papers for inclusion in the scoping review.
Participant or population: All studies that involved adult participants ( $\geq 18$ years old) with any degree or type of HL, tinnitus and/ or hyperacusis will be included in the review. Studies that included both children and adults will be excluded unless those two groups were assessed independently.

Intervention: Any study that determined MID for the outcomes of intervention for hearing disorders will be included. This includes, but is not limited to, surgery, HAs, noise generators and psychological therapies.

Comparator: Although not essential for this review, any study that includes comparators will be included.

Study designs to be included: This review will include published studies of any design. Grey literature (e.g. conference abstracts, doctoral theses, unpublished research, evaluation research) will be excluded to maintain the quality of the findings. No restriction will be applied in terms of language, geographic location or social, racial or gender demographics.

Eligibility criteria: The main eligibility criteria will be primary studies conducted on adults with hearing disorders (i.e., hearing loss, tinnitus and hyperacusis) that report minimal important difference.

Information sources: A medical information specialist searched the following electronic databases: PubMed, Web of Science, Psyclnfo, EMBASE (OVID), EMCARE (OVID), Cochrane Library, Academic Search Premier and the search engine of Google Scholar. Only the first 118 of 383 references in Google Scholar were extracted and will be screened for eligibility to decrease identifying irrelevant papers. There will be no publication date restriction to avoid excluding papers identified in non-indexed papers.

Main outcome(s): The primary outcome of interest, for which the MID has been determined using a well-defined approach (i.e. anchor-based, distribution-based or Delphi approaches), will be any self-report 
questionnaire, such as the Client-Oriented Scale of Improvement (Dillon et al., 1997) and the Tinnitus Functional Index (Meikle et al., 2012).

Additional outcome(s): Secondary outcomes of interest will be aided recognition tests, for example, hearing in noise tests (Nilsson et al., 1994) and hearing-specific quality of life, such as the Hearing Handicap Inventory for Adults (Newman et al., 1990). Any additional outcomes of interest, such as studies that used generic hearing-related quality of life or data-logging technology to estimate the efficacy of HAs', will also be included. The primary outcome of interest, for which the MID (using any of the recognised methods) has been determined, will be any selfreport questionnaire, such as the ClientOriented Scale of Improvement (Dillon et al., 1997) and the Tinnitus Functional Index (Meikle et al., 2012).

Data management: The data for each relevant publication will be imported into reference software (EndNote 20 Reference Manager, Clarivate, Luton Bedfordshire, UK). Prior to the initial screening, the same programme will be used to automatically delete any duplicate papers. Thereafter, author $A B$ will export the titles and abstracts of the selected papers into a spreadsheet (Excel version 2016, Microsoft Corporation, Redmont, WA). One author (AB) will screen the titles and abstracts of all the identified papers to assess their eligibility. A second reviewer (KJM) will screen a proportion of the identified records $(25 \%)$ to crosscheck the accuracy of the screening process. Any discrepancies in the data extraction will be resolved via discussion between both authors. A final determination will be made by consulting with a third author (IA) if no agreement is reached. Once a paper is selected based on the eligibility criteria, the full text of the paper will be read by $A B$. $A$ second author (KJM) will also read a proportion (25\%) of the full text to crosscheck the accuracy of the inclusion process. Any disagreement will be addressed through discussion or consultation with the third author (IA). The collected data from the studies that are included in the review will be presented using numerical (i.e. number and types of studies), narrative formats and tables. The summary report will discuss the implications of the findings for future research and practice. The Preferred Reporting Items for Systematic Review and Meta-Analyses Extension for Scoping Reviews (Tricco et al., 2018) flow chart will be applied to summarise the selection process to ensure transparency. This review will not draw any conclusions about the effectiveness of using MIDs with HA, tinnitus or hyperacusis outcome measurements among people with HL. A meta-analysis will therefore not be performed. Due to the broad scope of this review, the risk of bias will not be assessed to avoid potentially excluding manuscripts that examined MIDs in audiology. The extracted data will be summarised into four tables, one each for HL, tinnitus, hyperacusis and other types of hearing disorders (e.g. vestibular Schwannoma), as adapted from the Cochrane Handbook for Systematic Review of Interventions (Higgins and Green, 2011). The characteristics of the identified articles will be categorised as follows: (1) general information, (2) participant demographics, (3) method, (4) intervention, (5) outcome measurement, (6) MID approach and (7) funding and declaration of interest details (see Appendix III). AB will be responsible for the data extraction, and $30 \%$ of the extracted data will be evaluated by another researcher to ensure its accuracy.

Quality assessment / Risk of bias analysis: As scoping review research, there will be no formal quality assessment of studies.

Strategy of data synthesis: The data will be narratively synthesised.

Subgroup analysis: As scoping review research, there will be no plan for analysis subgroup data.

Sensitivity analysis: As scoping review research, there will be no plan to perform the sensitivity analysis of data. 
Language: No restrictions will be applied on language.

Country(ies) involved: United Kingdom, Saudi Arabia.

Keywords: Hearing disorders, hearing loss, tinnitus, hyperacusis, just-meaningful difference, minimally important differences, minimal clinically important difference, outcomes, scoping review.

Dissemination plans: The findings will be published in peer-reviewed journals and/or presented at scientific conferences.

Contributions of each author:

Author 1 - Abdullah Bin Shulhub - The author prepares and develops the protocol. The author will be included in the selection and data extraction process and will prepare the manuscript for this review.

Email: ashalhoob@ksu.edu.sa

Author 2 - Ibrahim Almufarrij - The author assisted in the creation of this manuscript, critically reviewed and provided thorough feedback on the previous versions. The author will also critically review the manuscript for this review.

Email: ibrahim.almufarrij@manchester.ac.uk Author 3 - Kevin J Munro - The author owns the research idea and has offered supervision of the research as well as feedback on its development. The author assisted in the creation of this manuscript, critically reviewed and provided thorough feedback on the previous versions. The author will also critically review the manuscript for this review.

Email: kevin.j.munro@manchester.ac.uk 